\title{
Treatment of Breast Cancer Brain Metastases through a Targeted Nanomolecule Drug Delivery System Based on Dopamine Functionalized Multi-Wall Carbon Nanotubes (MWCNTs) Coated with Nano Graphene Oxide (GO) and Protonated Polyaniline (PANI) in situ During the Polymerization of Aniline Autogenic Nanoparticles for the Delivery of Anti-Cancer Nano Drugs under Synchrotron Radiation
}

\author{
Alireza Heidari* \\ Faculty of Chemistry, California South University, 14731 Comet St. Irvine, CA 92604, USA
}

${ }^{*}$ Corresponding Author: Prof. Dr. Alireza Heidari, Faculty of Chemistry, California South University (CSU), 14731 Comet St. Irvine, CA 92604, USA, Tel: +1-775-410-4974; E-mail: Scholar.Researcher.Scientist@gmail.com

Received Date: July 03, 2017; Accepted Date: July 05, 2017; Published Date: July 08, 2017

Copyright: (ㄷ 2017 Heidari A. This is an open-access article distributed under the terms of the Creative Commons Attribution License, which permits unrestricted use, distribution, and reproduction in any medium, provided the original author and source are credited.

Citation: Heidari A. Treatment of Breast Cancer Brain Metastases through a Targeted Nanomolecule Drug Delivery System Based on Dopamine Functionalized Multi-Wall Carbon Nanotubes (MWCNTs) Coated with Nano Graphene Oxide (GO) and Protonated Polyaniline (PANI) in situ During the Polymerization of Aniline Autogenic Nanoparticles for the Delivery of Anti-Cancer Nano Drugs under Synchrotron Radiationt. Br J Res 2017, 4(3): 16.

\section{Editorial}

In the last few years, treatment of breast cancer brain metastases through a targeted Nanomolecule drug delivery system based on dopamine functionalized Multi-Wall Carbon Nanotubes (MWCNTs) coated with Nano Graphene Oxide (GO) and protonated Polyaniline (PANI) in situ during the polymerization of aniline autogenic nanoparticles for the delivery of anti-cancer Nano drugs under synchrotron radiation was of special interest in various industrial and scientific fields, and also in many area of pharmaceutical and medicinal chemistry involving different catalytic processes [1-73]. The enzymatic determination of dopamine is particularly needed in pharmaceutical and medicinal antiseptic or disinfectant samples, cosmetic bleach hair agent, and also in the plastic industry to make polymers. In very concentrated solution (98\%), it has also been used as an oxidizer for rocket propulsion. In this editorial, the chemometric full factorial design analysis was used to treatment of breast cancer brain metastases through a targeted Nanomolecule drug delivery system based on dopamine functionalized Multi-Wall Carbon Nanotubes (MWCNTs) coated with Nano Graphene Oxide (GO) and protonated Polyaniline (PANI) in situ during the polymerization of aniline autogenic nanoparticles for the delivery of anti-cancer Nano drugs under synchrotron radiation. The enzymatic reaction was optimized by combining an electrokinetic method with a fluoride ion selective electrode in two steps, where the range of critical variables was first determined based on a preliminary "one-variable-at-atime" (OVAT) procedure for the subsequent full factorial design chemometric analysis. As a result, the impact of the experimental factors, their interactions and also their optimum values were evaluated by the statistical analysis of variance (ANOVA). The optimized model was validated by the assay of dopamine in real samples.
The enzymatic hydrolysis reaction of urea by urease is optimized in this editorial work using an initial rate potentiometric method and the chemometric Response Surface Methodology (RSM). The optimization was based by combining "one-variable-at-a-time" (OVAT) procedure with Response Surface Methodology (RSM) chemometric analysis. As a result, the non-linear nature of the experimental response of the enzymatic reaction system was satisfactory explained by a second order polynomial equation, which revealed the impact of the experimental factors, their interactions and also their optimum values. The results of the reported Response Surface Methodology (RSM) analysis proved; also, to be quite appropriate for the design and optimization of the hydrolysis reaction as illustrated by the relatively high value of the determination coefficient generated by fitting of the quadratic model, along with the satisfactory results obtained by the analysis of variance (ANOVA). In addition, in order to check the quality of the optimization and the validity of the model, the assay of urea, both in aqueous laboratory and human serum samples were performed for treatment of breast cancer brain metastases through a targeted Nanomolecule drug delivery system based on dopamine functionalized Multi-Wall Carbon Nanotubes (MWCNTs) coated with Nano Graphene Oxide (GO) and protonated Polyaniline (PANI) in situ during the polymerization of aniline autogenic nanoparticles for the delivery of anti-cancer Nano drugs under synchrotron radiation.

\section{References}

1. Heidari A, Brown C (2015) Study of Composition and Morphology of Cadmium Oxide (CdO) Nanoparticles for Eliminating Cancer Cells. J Nanomed Res 2: 20.

2. Heidari A, Brown C (2015) Study of Surface Morphological, Phytochemical and Structural Characteristics of Rhodium (III) 
Oxide (Rh203) Nanoparticles. Int J Pharmacol Phytochem Ethnomed 1: 15-19.

3. Heidari A (2016) An Experimental Biospectroscopic Study on Seminal Plasma in Determination of Semen Quality for Evaluation of Male Infertility. Int J Adv Technol 7: e007.

4. Heidari A (2016) Extraction and Preconcentration of N-TolylSulfonyl-Phosphoramid-Saeure-Dichlorid as an Anti-Cancer Drug from Plants: A Pharmacognosy Study. J Pharmacogn Nat Prod 2: e103.

5. Heidari A (2016) A Thermodynamic Study on Hydration and Dehydration of DNA and RNA-Amphiphile Complexes. J Bioeng Biomed Sci S: 006.

6. Heidari A (2016) Computational Studies on Molecular Structures and Carbonyl and Ketene Groups' Effects of Singlet and Triplet Energies of Azidoketene $\mathrm{O}=\mathrm{C}=\mathrm{CH}-\mathrm{NNN}$ and Isocyanatoketene $\mathrm{O}=\mathrm{C}=\mathrm{CH}-\mathrm{N}=\mathrm{C}=\mathrm{O}$. J Appl Computat Math 5: e142.

7. Heidari A (2016) Study of Irradiations to Enhance the Induces the Dissociation of Hydrogen Bonds between Peptide Chains and Transition from Helix Structure to Random Coil Structure Using ATR-FTIR, Raman and 1HNMR Spectroscopies. J Biomol Res Ther 5: e146.

8. Heidari A (2016) Future Prospects of Point Fluorescence Spectroscopy, Fluorescence Imaging and Fluorescence Endoscopy in Photodynamic Therapy (PDT) for Cancer Cells. J Bioanal Biomed 8: e135.

9. Heidari A (2016) A Bio-Spectroscopic Study of DNA Density and Color Role as Determining Factor for Absorbed Irradiation in Cancer Cells. Adv Cancer Prev 1: e102.

10. Heidari A (2016) Manufacturing Process of Solar Cells Using Cadmium Oxide (CdO) and Rhodium (III) Oxide (Rh2O3) Nanoparticles. J Biotechnol Biomater 6: e125.

11. Heidari A (2016) A Novel Experimental and Computational Approach to Photobiosimulation of Telomeric DNA/RNA: A Biospectroscopic and Photobiological Study. J Res Development 4: 144.

12. Heidari A (2016) Biochemical and Pharmacodynamical Study of Microporous Molecularly Imprinted Polymer Selective for Vancomycin, Teicoplanin, Oritavancin, Telavancin and Dalbavancin Binding, Biochem Physiol 5: e146.

13. Heidari A (2016) Anti-Cancer Effect of UV Irradiation at Presence of Cadmium Oxide (CdO) Nanoparticles on DNA of Cancer Cells: A Photodynamic Therapy Study. Arch Cancer Res 4: 1.

14. Heidari A (2016) Biospectroscopic Study on Multi-Component Reactions (MCRs) in Two A-Type and B-Type Conformations of Nucleic Acids to Determine Ligand Binding Modes, Binding Constant and Stability of Nucleic Acids in Cadmium Oxide (CdO) Nanoparticles-Nucleic Acids Complexes as Anti-Cancer Drugs. Arch Cancer Res 4: 2.

15. Heidari A (2016) Simulation of Temperature Distribution of DNA/RNA of Human Cancer Cells Using Time- Dependent BioHeat Equation and Nd: YAG Lasers. Arch Cancer Res 4: 2.

16. Heidari A (2016) Quantitative Structure-Activity Relationship (QSAR) Approximation for Cadmium Oxide (CdO) and Rhodium (III) Oxide (Rh2O3) Nanoparticles as Anti-Cancer Drugs for the Catalytic Formation of Proviral DNA from Viral RNA Using Multiple Linear and Non-Linear Correlation Approach. Ann Clin Lab Res 4: 1.
17. Heidari A (2016) Biomedical Study of Cancer Cells DNA Therapy Using Laser Irradiations at Presence of Intelligent Nanoparticles. J Biomedical Sci 5: 2.

18. Heidari A (2016) Measurement the Amount of Vitamin D2 (Ergocalciferol), Vitamin D3 (Cholecalciferol) and Absorbable Calcium (Ca2+), Iron (II) (Fe2+), Magnesium (Mg2+), Phosphate (PO4-) and Zinc (Zn2+) in Apricot Using High-Performance Liquid Chromatography (HPLC) and Spectroscopic Techniques. J Biom Biostat 7: 292

19. Heidari A (2016) Spectroscopy and Quantum Mechanics of the Helium Dimer $(\mathrm{He} 2+)$, Neon Dimer (Ne2+), Argon Dimer (Ar2+), Krypton Dimer (Kr2+), Xenon Dimer (Xe2+), Radon Dimer(Rn2+) and Ununoctium Dimer (Uuo2+) Molecular Cations. Chem Sci J 7: e112.

20. Heidari A (2016) Human Toxicity Photodynamic Therapy Studies on DNA/RNA Complexes as a Promising New Sensitizer for the Treatment of Malignant Tumors Using Bio-Spectroscopic Techniques. J Drug Metab Toxicol 7: e129.

21. Heidari A (2016) Novel and Stable Modifications of Intelligent Cadmium Oxide (CdO) Nanoparticles as Anti-Cancer Drug in Formation of Nucleic Acids Complexes for Human Cancer Cells' Treatment. Biochem Pharmacol (Los Angel) 5: 207.

22. Heidari A (2016) A Combined Computational and $Q M / M M$ Molecular Dynamics Study on Boron Nitride Nanotubes (BNNTs), Amorphous Boron Nitride Nanotubes (a-BNNTs) and Hexagonal Boron Nitride Nanotubes (h-BNNTs) as Hydrogen Storage. Struct Chem Crystallogr Commun 2: 1.

23. Heidari A (2016) Pharmaceutical and Analytical Chemistry Study of Cadmium Oxide (CdO) Nanoparticles Synthesis Methods and Properties as Anti-Cancer Drug and its Effect on Human Cancer Cells. Pharm Anal Chem Open Access 2: 113.

24. Heidari A (2016) A Chemotherapeutic and Biospectroscopic Investigation of the Interaction of Double-Standard DNA/RNABinding Molecules with Cadmium Oxide (CdO) and Rhodium (III) Oxide (Rh2O3) Nanoparticles as Anti-Cancer Drugs for Cancer Cells' Treatment. Chemo Open Access 5: e129.

25. Heidari A ( 2016) Pharmacokinetics and Experimental Therapeutic Study of DNA and Other Biomolecules Using Lasers: Advantages and Applications. J Pharmacokinet Exp Ther 1: e005

26. Heidari A (2016) Determination of Ratio and Stability Constant of DNA/RNA in Human Cancer Cells and Cadmium Oxide (CdO) Nanoparticles Complexes Using Analytical Electrochemical and Spectroscopic Techniques. Insights Anal Electrochem 2: 1.

27. Heidari A (2016) Discriminate between Antibacterial and NonAntibacterial Drugs Artificial Neutral Networks of a Multilayer Perceptron (MLP) Type Using a Set of Topological Descriptors. J Heavy Met Toxicity Dis 1: 2.

28. Heidari A (2016) Combined Theoretical and Computational Study of the Belousov-Zhabotinsky Chaotic Reaction and Curtius Rearrangement for Synthesis of Mechlorethamine, Cisplatin, Streptozotocin, Cyclophosphamide, Melphalan, Busulphan and BCNU as Anti-Cancer Drugs. Insights Med Phys 1: 2.

29. Heidari A (2016) A Translational Biomedical Approach to Structural Arrangement of Amino Acids' Complexes: A Combined Theoretical and Computational Study. Transl Biomed 7: 2.

30. Heidari A (2016) Ab Initio and Density Functional Theory (DFT) Studies of Dynamic NMR Shielding Tensors and Vibrational Frequencies of DNA/RNA and Cadmium Oxide (CdO) 
Nanoparticles Complexes in Human Cancer Cells. J Nanomedine Biotherapeutic Discov 6: e144.

31. Heidari A (2016) Molecular Dynamics and Monte-Carlo Simulations for Replacement Sugars in Insulin Resistance, Obesity, LDL Cholesterol, Triglycerides, Metabolic Syndrome, Type 2 Diabetes and Cardiovascular Disease: A Glycobiological Study. J Glycobiol 5: e111.

32. Heidari A (2016) Synthesis and Study of 5[(Phenylsulfonyl)Amino]-1,3,4-Thiadiazole-2-Sulfonamide as Potential Anti-Pertussis Drug Using Chromatography and Spectroscopy Techniques. Transl Med (Sunnyvale) 6:e138.

33. Heidari A (2016) Nitrogen, Oxygen, Phosphorus and Sulphur Heterocyclic Anti-Cancer Nano Drugs Separation in the Supercritical Fluid of Ozone (O3) Using Soave-Redlich-Kwong (SRK) and Pang-Robinson (PR) Equations. Electronic J Biol 12:4.

34. Heidari A (2016) An Analytical and Computational Infrared Spectroscopic Review of Vibrational Modes in Nucleic Acids. Austin J Anal Pharm Chem 3:1058.

35. Heidari A, Brown C (2016) Phase, Composition and Morphology Study and Analysis of Os-Pd/HfC Nanocomposites. Nano Res Appl 2: 1 .

36. Heidari A, Brown C (2016) Vibrational Spectroscopic Study of Intensities and Shifts of Symmetric Vibration Modes of Ozone Diluted by Cumene. Int J Advanced Chem 4: 5-9.

37. Heidari A (2016) Study of the Role of Anti-Cancer Molecules with Different Sizes for Decreasing Corresponding Bulk Tumor Multiple Organs or Tissues. Arch Can Res 4: 2.

38. Heidari A (2016) Genomics and Proteomics Studies of Zolpidem, Necopidem, Alpidem, Saripidem, Miroprofen, Zolimidine, Olprinone and Abafungin as Anti-Tumor, Peptide Antibiotics, Antiviral and Central Nervous System (CNS) Drugs. J Data Mining Genomics \& Proteomics 7: e125.

39. Heidari A (2016) Pharmacogenomics and Pharmacoproteomics Studies of Phosphodiesterase-5 (PDE5) Inhibitors and Paclitaxel Albumin-Stabilized Nanoparticles as Sandwiched Anti-Cancer Nano Drugs between Two DNA/RNA Molecules of Human Cancer Cells. J Pharmacogenomics Pharmacoproteomics 7: e153.

40. Heidari A (2016) Biotranslational Medical and Biospectroscopic Studies of Cadmium Oxide (CdO) Nanoparticles-DNA/RNA Straight and Cycle Chain Complexes as Potent Anti-Viral, Anti-Tumor and Anti-Microbial Drugs: A Clinical Approach. Transl Biomed 7: 2.

41. Heidari A (2016) A Comparative Study on Simultaneous Determination and Separation of Adsorbed Cadmium Oxide (CdO) Nanoparticles on DNA/RNA of Human Cancer Cells Using Biospectroscopic Techniques and Dielectrophoresis (DEP) Method. Arch Can Res 4: 2.

42. Heidari A (2016) Cheminformatics and System Chemistry of Cisplatin, Carboplatin, Nedaplatin, Oxaliplatin, Heptaplatin and Lobaplatin as Anti-Cancer Nano Drugs: A Combined Computational and Experimental Study. J Inform Data Min 1: 3.

43. Heidari A (2016) Linear and Non-Linear Quantitative StructureAnti-Cancer-Activity Relationship (QSACAR) Study of Hydrous Ruthenium (IV) Oxide (RuO2) Nanoparticles as Non-Nucleoside Reverse Transcriptase Inhibitors (NNRTIs) and Anti-Cancer Nano Drugs. J Integr Oncol 5: e110.

44. Heidari A (2016) Synthesis, Characterization and Biospectroscopic Studies of Cadmium Oxide (CdO) Nanoparticles- Nucleic Acids Complexes Absence of Soluble Polymer as a Protective Agent
Using Nucleic Acids Condensation and Solution Reduction Method. J Nanosci Curr Res 1: e101.

45. Heidari A (2016) Coplanarity and Collinearity of 4'-Dinonyl-2,2'Bithiazole in One Domain of Bleomycin and Pingyangmycin to be Responsible for Binding of Cadmium Oxide (CdO) Nanoparticles to DNA/RNA Bidentate Ligands as Anti-Tumor Nano Drug. Int J Drug Dev \& Res 8: 007-008.

46. Heidari A (2016) A Pharmacovigilance Study on Linear and NonLinear Quantitative Structure (Chromatographic) Retention Relationships (QSRR) Models for the Prediction of Retention Time of Anti-Cancer Nano Drugs under Synchrotron Radiations. J Pharmacovigil 4: e161.

47. Heidari A (2016) Nanotechnology in Preparation of Semipermeable Polymers. J Adv Chem Eng 6: 157.

48. Heidari A (2016) A Gastrointestinal Study on Linear and NonLinear Quantitative Structure (Chromatographic) Retention Relationships (QSRR) Models for Analysis 5-Aminosalicylates Nano Particles as Digestive System Nano Drugs under Synchrotron Radiations .J Gastrointest Dig Syst 6: e119.

49. Heidari A (2016) DNA/RNA Fragmentation and Cytolysis in Human Cancer Cells Treated with Diphthamide Nano Particles Derivatives. Biomedical Data Mining 5: e102.

50. Heidari A (2016) A Successful Strategy for the Prediction of Solubility in the Construction of Quantitative Structure-Activity Relationship (QSAR) and Quantitative Structure-Property Relationship (QSPR) under Synchrotron Radiations Using Genetic Function Approximation (GFA) Algorithm. J Mol Biol Biotechnol 1: 1.

51. Heidari A (2016) Computational Study on Molecular Structures of C20, C60, C240, C540, C960, C2160 and C3840 Fullerene Nano Molecules under Synchrotron Radiations Using Fuzzy Logic. J Material Sci Eng 5: 282.

52. Heidari A (2016) Graph Theoretical Analysis of Zigzag Polyhexamethylene Biguanide, Polyhexamethylene Adipamide, Polyhexamethylene Biguanide Gauze and Polyhexamethylene Biguanide Hydrochloride (PHMB) Boron Nitride Nanotubes (BNNTs), Amorphous Boron Nitride Nanotubes (a-BNNTs) and Hexagonal Boron Nitride Nanotubes (h-BNNTs). J Appl Computat Math 5: e143.

53. Heidari A (2016) The Impact of High Resolution Imaging on Diagnosis. Int J Clin Med Imaging 3: 1000e101.

54. Heidari A (2016) A Comparative Study of Conformational Behavior of Isotretinoin (13-Cis Retinoic Acid) and Tretinoin (All-Trans Retinoic Acid (ATRA)) Nano Particles as Anti-Cancer Nano Drugs under Synchrotron Radiations Using Hartree-Fock (HF) and Density Functional Theory (DFT) Methods. Insights in Biomed 1: 2.

55. Heidari A (2016) Advances in Logic, Operations and Computational Mathematics. J Appl Computat Math 5: 5, 2016.

56. Heidari A (2016) Mathematical Equations in Predicting Physical Behavior. J Appl Computat Math 5: 5.

57. Heidari A (2016) Chemotherapy a Last Resort for Cancer Treatment. Chemo Open Access 5: 4.

58. Heidari A (2016) Separation and Pre-Concentration of Metal Cations-DNA/RNA Chelates Using Molecular Beam Mass Spectrometry with Tunable Vacuum Ultraviolet (VUV) Synchrotron Radiation and Various Analytical Methods. Mass Spectrom Purif Tech 2: e101.

59. Heidari A (2016) Yoctosecond Quantitative Structure-Activity Relationship (QSAR) and Quantitative Structure- Property 
Relationship (QSPR) under Synchrotron Radiations Studies for Prediction of Solubility of Anti-Cancer Nano Drugs in Aqueous Solutions Using Genetic Function Approximation (GFA) Algorithm. Insight Pharm Res. 1: 1.

60. Heidari A (2016) Cancer Risk Prediction and Assessment in Human Cells under Synchrotron Radiations Using Quantitative Structure Activity Relationship (QSAR) and Quantitative Structure Properties Relationship (QSPR) Studies. Int J Clin Med Imaging 3: 516.

61. Heidari A (2016) A Novel Approach to Biology. Electronic J Biol 12 : 4.

62. Heidari A (2016) Innovative Biomedical Equipment's for Diagnosis and Treatment. J Bioengineer \& Biomedical Sci 6: 2.

63. Heidari A (2016) Integrating Precision Cancer Medicine into Healthcare, Medicare Reimbursement Changes and the Practice of Oncology: Trends in Oncology Medicine and Practices. J Oncol Med \& Pract 1: 2

64. Heidari A (2016) Promoting Convergence in Biomedical and Biomaterials Sciences and Silk Proteins for Biomedical and Biomaterials Applications: An Introduction to Materials in Medicine and Bioengineering Perspectives. J Bioengineer \& Biomedical Sci 6: 3.

65. Heidari A (2017) X-Ray Fluorescence and X-Ray Diffraction Analysis on Discrete Element Modeling of Nano Powder Metallurgy Processes in Optimal Container Design. J Powder Metall Min 6: 1

66. Heidari A (2017) Biomolecular Spectroscopy and Dynamics of Nano-Sized Molecules and Clusters as Cross- Linking-Induced Anti-Cancer and Immune-Oncology Nano Drugs Delivery in DNA/RNA of Human Cancer Cells' Membranes under Synchrotron Radiations: A Payload-Based Perspective. Arch Chem Res 1: 2.

67. Heidari A (2017) Deficiencies in Repair of Double-Standard DNA/ RNA-Binding Molecules Identified in Many Types of Solid and
Liquid Tumors Oncology in Human Body for Advancing Cancer Immunotherapy Using Computer Simulations and Data Analysis. J Appl Bioinforma Comput Biol 6: 1.

68. Heidari A (2017) Electronic Coupling among the Five Nanomolecules Shuts Down Quantum Tunneling in the Presence and Absence of an Applied Magnetic Field for Indication of the Dimer or other Provide Different Influences on the Magnetic Behavior of Single Molecular Magnets (SMMs) as Qubits for Quantum Computing. Glob J Res Rev 4: 2.

69. Heidari A (2017). Polymorphism in Nano-Sized Graphene LigandInduced Transformation of Au38-xAgx/xCux(SPh- tBu)24 to Au36-xAgx/xCux(SPh-tBu)24 (x=1-12) Nanomolecules for Synthesis of Au144-xAgx/xCux[(SR)60, (SC4)60, (SC6)60, (SC12)60, (PET)60, (p-MBA)60, (F)60, (Cl)60, (Br)60, (I)60, (At)60, (Uus)60 and (SC6H13)60] Nano Clusters as Anti-Cancer Nano Drugs. J Nanomater Mol Nanotechnol 6: 3.

70. Heidari A (2017) Biomedical Resource Oncology and Data Mining to Enable Resource Discovery in Medical, Medicinal, Clinical, Pharmaceutical, Chemical and Translational Research and Their Applications in Cancer Research. Int J Biomed Data Min 6: e103.

71. Heidari A. Study of Synthesis, Pharmacokinetics, Pharmacodynamics, Dosing, Stability, Safety and Efficacy of Olympiadane Nanomolecules as Agent for Cancer Enzymotherapy, Immunotherapy, Chemotherapy, Radiotherapy, Hormone Therapy and Targeted Therapy under Synchrotorn Radiation. J Dev Drugs 6: e154.

72. Heidari A (2017) A Novel Approach to Future Horizon of Top Seven Biomedical Research Topics to Watch in 2017: Alzheimer's, Ebola, Hypersomnia, Human Immunodeficiency Virus (HIV), Tuberculosis (TB), Microbiome/Antibiotic Resistance and Endovascular Stroke. J Bioengineer \& Biomedical Sci 7: e127.

73. Heidari A (2017) Opinion on Computational Fluid Dynamics (CFD) Technique. Fluid Mech Open Acc 4: 157. 